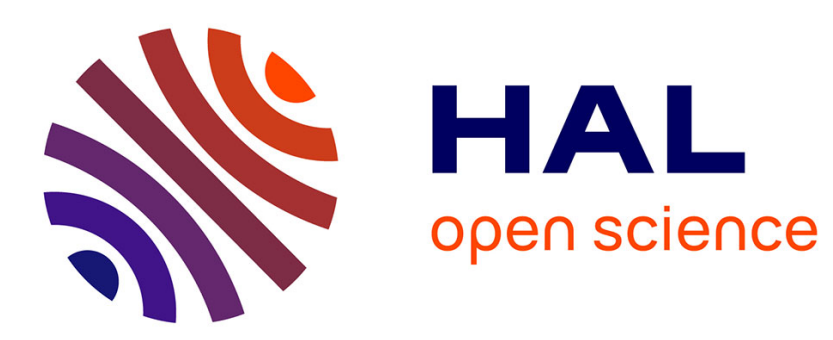

\title{
The Aperiodic Double Chain Model Applied to Composition Flexible Compounds
}

\author{
Ted Janssen, Juan Manuel Pérez-Mato
}

\section{To cite this version:}

Ted Janssen, Juan Manuel Pérez-Mato. The Aperiodic Double Chain Model Applied to Composition Flexible Compounds. Philosophical Magazine, 2007, 87 (18-21), pp.3031-3041. 10.1080/14786430701355125 . hal-00513831

\section{HAL Id: hal-00513831 \\ https://hal.science/hal-00513831}

Submitted on 1 Sep 2010

HAL is a multi-disciplinary open access archive for the deposit and dissemination of scientific research documents, whether they are published or not. The documents may come from teaching and research institutions in France or abroad, or from public or private research centers.
L'archive ouverte pluridisciplinaire HAL, est destinée au dépôt et à la diffusion de documents scientifiques de niveau recherche, publiés ou non, émanant des établissements d'enseignement et de recherche français ou étrangers, des laboratoires publics ou privés. 


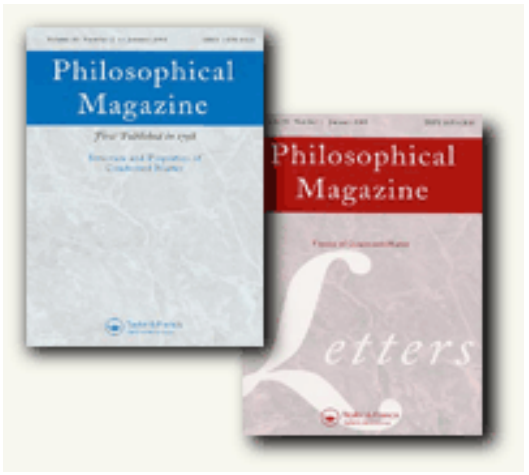

\section{The Aperiodic Double Chain Model Applied to Composition Flexible Compounds}

\begin{tabular}{|r|l|}
\hline Journal: & Philosophical Magazine \& Philosophical Magazine Letters \\
\hline Manuscript ID: & TPHM-06-Aug-0334.R1 \\
\hline Journal Selection: & Philosophical Magazine \\
\hline Author: & 09-Dec-2006 \\
\hline Complete List of Authors: & $\begin{array}{l}\text { Janssen, Ted; University of Nijmegen, Theoretical Physics } \\
\text { Pérez-Mato, Juan Manuel; Universidad del País Vasco, Física de } \\
\text { Kateria Condensada }\end{array}$ \\
\hline \hline & composite materials, incommensurate structures, lattice dynamics \\
\hline Keywords (user supplied): & $\begin{array}{l}\text { incommensurate composite, composition flexible compounds, } \\
\text { composite modelling }\end{array}$ \\
\hline \hline & \\
\hline $\begin{array}{l}\text { Note: The following files were submitted by the author for peer review, but cannot be converted } \\
\text { to PDF. You must view these files (e.g. movies) online. }\end{array}$ \\
\hline $\begin{array}{l}\text { refaper06.bib } \\
\text { Aperiodic06_jape_new.tex }\end{array}$
\end{tabular}

\section{今scholarONE \\ Manuscript Central}




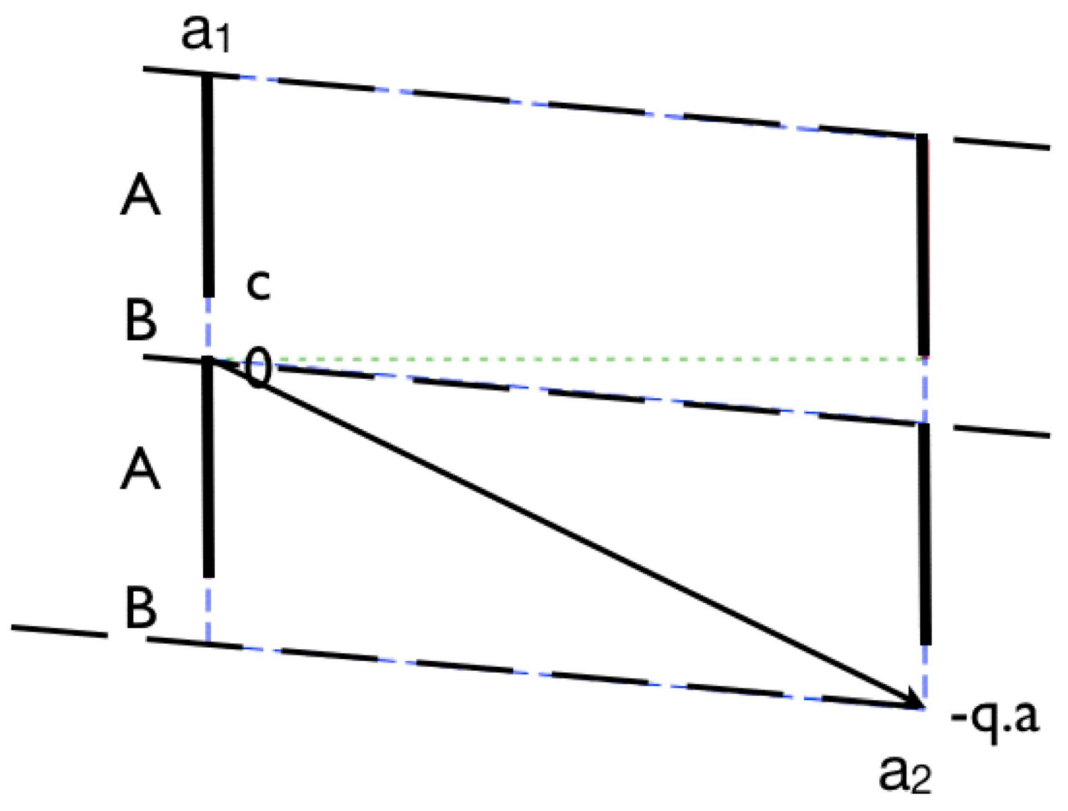

Figure 1 $237 \times 176 \mathrm{~mm}(300 \times 300 \mathrm{DPI})$ 
Philosophical Magazine \& Philosophical Magazine Letters

Page 2 of 11

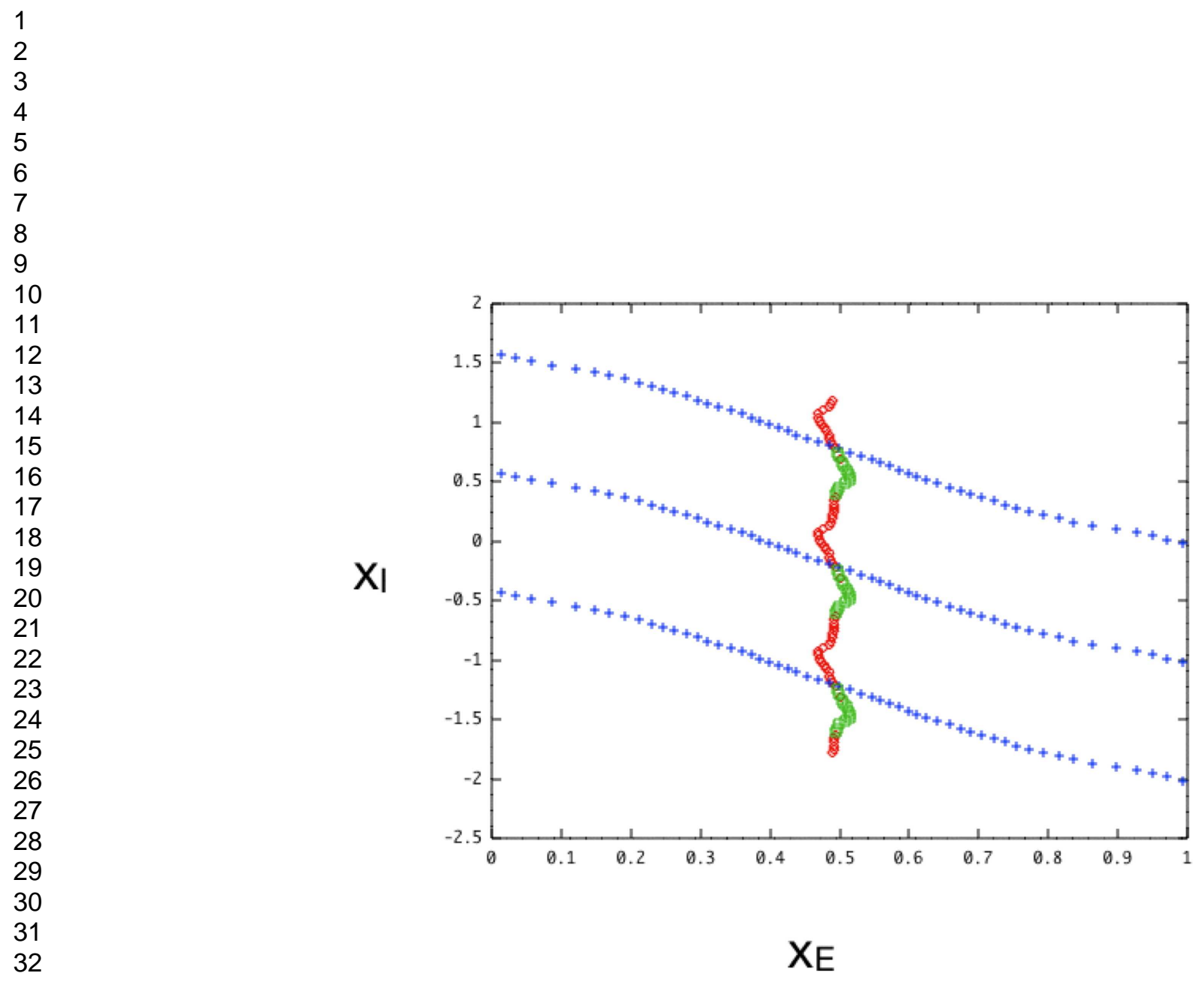

Figure 2

$217 \times 173 \mathrm{~mm}(300 \times 300$ DPI $)$

http://mc.manuscriptcentral.com/pm-pml 


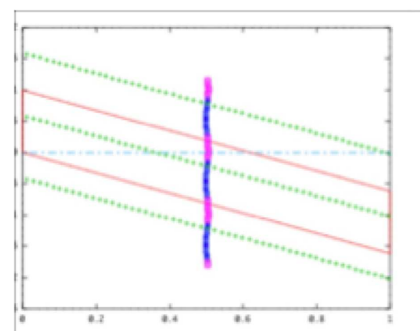

Weak int., uniform $\Delta=0$

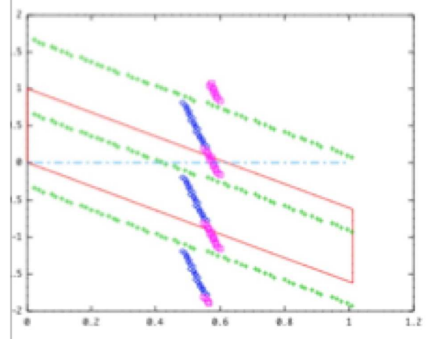

$\Delta=0.05$, weakly uniform

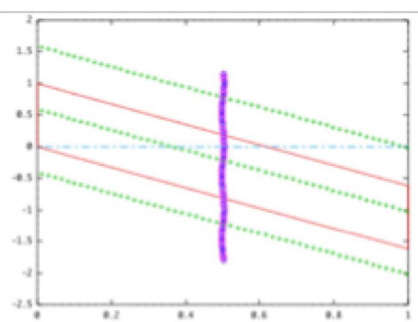

uniform internal

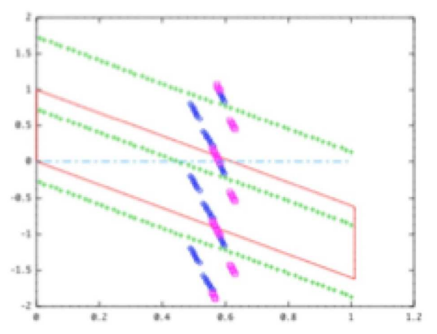

stronger non-uniform

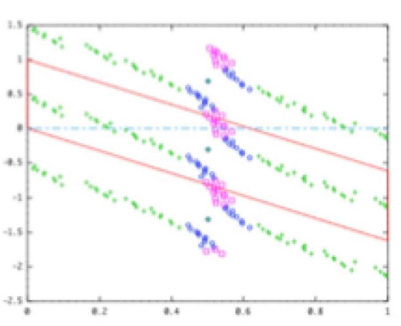

strong interaction

Figure 3

$246 \times 190 \mathrm{~mm}(300 \times 300$ DPI $)$ 


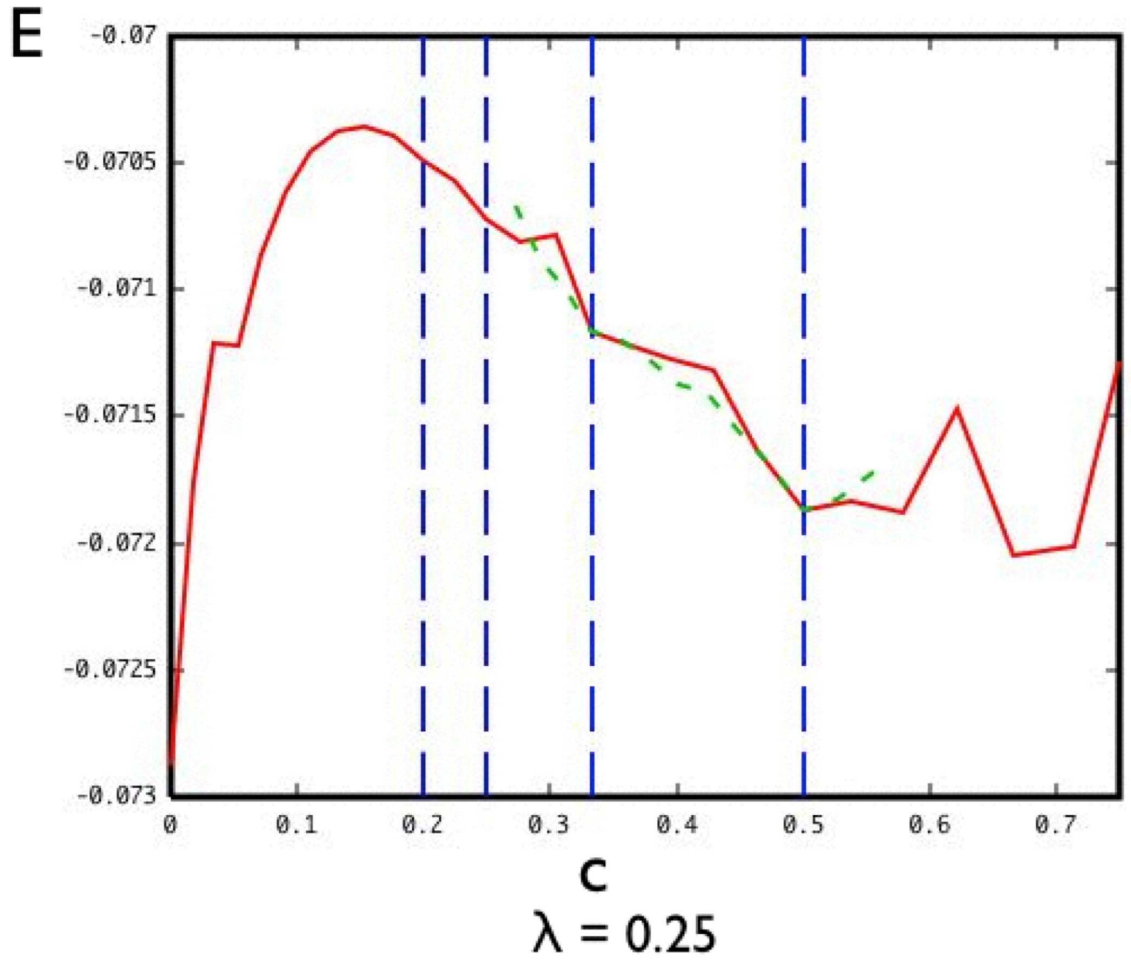

Figure 4

$203 \times 175 \mathrm{~mm}(300 \times 300 \mathrm{DPI})$

http://mc.manuscriptcentral.com/pm-pml 
$\omega$
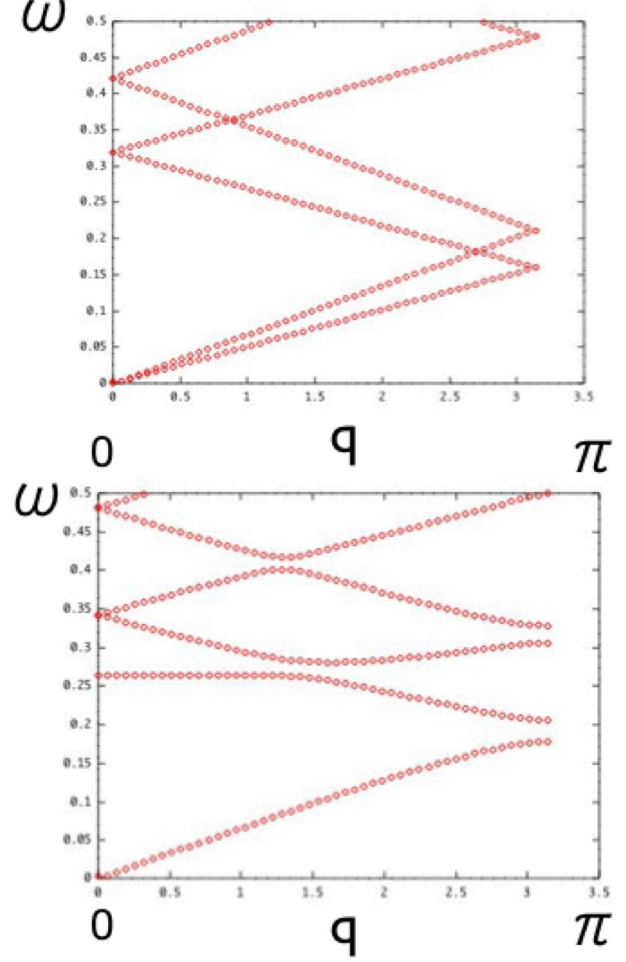

$\omega$

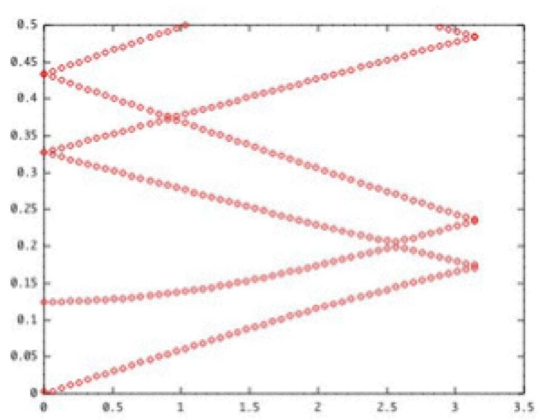

(u)

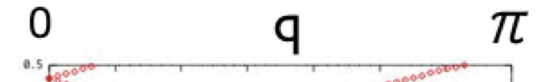

$\omega$

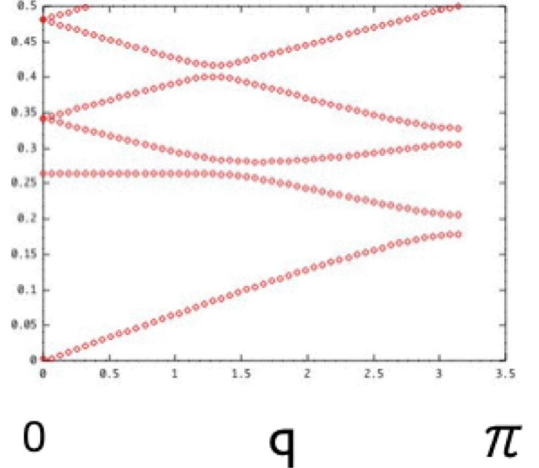

Figure 5

$254 \times 203 \mathrm{~mm}(300 \times 300 \mathrm{DPI})$ 

Applied to Composition Flexible Compounds

16 simple model for composition flexible compounds is studied. A discussion is given of the coupling between structure and composition, 1 and of the dynamics.

T. Janssen, Institute for Theoretical Physics, University of Nijmegen,6525ED Nijmegen, The Netherlands J.-M. Perez-Mato, Dept. Fisica de la Materia Condensada, Universidad del Pais Vasco, Bilbao, Spain

(Received 00 Month 200x; in final form 00 Month 200x)

22 he family of compounds, related to hexagonal perovskites, and with formula $A_{1+x}\left(A_{x}^{\prime} B_{1-x}\right) O_{3}[2,5]$ may be described as incommen23 rate composites. One subsystem consists of incommensurate columns of octahedra and triangular prisms. In between there are chains 24 atoms. The parameters are the composition $c$, related to the modulation wave vector $q$ and the height difference $2 \Delta$ between an 25 tahedron and a prism. The rank of the system is four: the wave vector of the $\left(A^{\prime}, B\right) O_{3}$ column and the mismatch parameter between the two subsystems are related. The embedding of the structure has finite, disjoint atomic surfaces, which means that the compounds 26 as well be described as modulated phases with an occupation modulation (with a crenel modulation function) and a displacive 277odulation (saw-tooth modulation function) [1].

28 Incommensurate composites have been studied with the Double Chain Model (DCM) [6]. It is a system consisting of two parallel 29ains at a distance $d$, where the lattice constant $a$ of the first chain is incommensurate with the lattice constant $b$ of the second. The 30 tra-chain interaction is limited to nearest neighbours. The potential energy is given by

$$
V=\sum_{n} V_{1}\left(x_{n}-x_{n-1}\right)+\sum_{m} V_{2}\left(y_{m}-y_{m-1}\right)+\lambda \sum_{n m} V_{i}\left(x_{n}, y_{m}\right) .
$$

33

34

$53^{3}$ is incommensurate because $a / b$ is supposed to be irrational, and is approximated by the fraction $n_{2} / n_{1}$.

53 For this model the ground state and the excitations have been calculated numerically. For the interaction parameter $\lambda=0$ $54_{1}$ e chains are unmodulated, for larger values they are modulated, with continuous modulation functions for $\lambda<\lambda_{c}$ and discontinuous 55odulation functions if $\lambda>\lambda_{c}$. For $\lambda<\lambda_{c}$, there are two vibration modes with frequency equal to zero, an acoustic and a phason mode. 56 the other case, there is only one such mode, the acoustic mode, and the phason branch has a gap.

57 A generalisation gives the aperiodic double chain model (APDC). In this model one of the subsystems has a quasiperiodic 57 erage structure. An example is a harmonic chain in combination with a Fibonacci chain. When there are no special relations between 58 e modulation wave vector and the reciprocal lattice of the second chain, the rank of the Fourier module is three or more. It is similar 59 a generalised Frenkel-Kontorova model [7]. 
Philosophical Magazine,

10ol. 00, No. 00, DD Month 200x, 2-6

The model

For the composition flexible compounds we may use this APDC as well. The chain corresponding to the system of octahedra and prisms 4has an incommensurability which has its origin in the coupling to the other chain. However, there are two types of incommensurability 5ere. One is the sequence of prisms and octahedra, which is a discrete sequence, and one is the displacements wave induced by the interaction. For given sequence of building blocks this may be modelled by the APDC model.

The second chain now represents the atoms between the stacks of octahedra and prisms. They form a modulated chain with

7average distance $b$. The first chain represents the stacks and is composed of $n_{11}$ intervals of length $a_{1}$ and $n_{12}$ intervals of length $a_{2}$. The gength, the difference between the average positions $\bar{x}_{n}$ and $\bar{x}_{n-1}$, corresponds to the value of a spin variable $\sigma_{n}$ such that

9

10

$$
\bar{x}_{n}-\bar{x}_{n-1}=a+\sigma_{n} \Delta .
$$

$12^{11}$

13

14

15

16

17

18

1 Fom this follows that

20

21

22

2Botice that, for $\Delta=0$, we recover the original DCM.

24 The potential energy of the systems becomes

25

26

27

28

Here $V_{1}(x, \sigma)=\alpha(x-a-\sigma \Delta)^{2} / 2$ and $V_{2}(y)=\beta y^{2} / 2$. In principle the interaction $V_{i}$ also depends on $\sigma$. This is taken into account by Considering as variation parameter the discrete set of spin configurations $\sigma$. The influence of $\sigma$ on the inter-chain interaction (intervals 30 different length correspond actually to different atoms) is neglected here. By the interaction the second chain becomes displacively 3nodulated, the first chain has an aperiodic average structure which also becomes modulated by the interaction. The average distance of

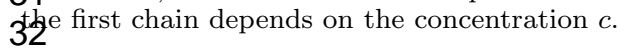

33

34

35 36 38 jections of the basis vectors $(1 / \bar{a}, 0)$ and $(1 / b, 1)$. The corresponding basis for the direct lattice consists of $(\bar{a},-\bar{a} / b)$ and $(0,1)$. The 39 commensurability is given by $\bar{a} / b=n_{2} / n_{1}$.

40

41

42

43

44

45

46

47

48

49

50

51

52

53 Figure 1. Embedding of the system, and closeness condition. The vertical lines correspond to spin up of chain 1 , the gaps to spin 5 \$own. The dashed line corresponds to the positions of chain 2 . The vectors $\mathbf{a}_{1}$ and $\mathbf{a}_{2}$ span the unit cell. The upper end of the spin up part in one atomic surface corresponds to the lower end in a neighbouring atomic surface.

The above mentioned relation between the modulation wave vector $q$ and the concentration $c$ is given by the so called closeness 59 ndition. The closeness condition says that the projection of the boundary of an atomic surface should coincide with that of another atomic surface. The term is usually used in the description of quasicrystals, but it is a general concept for quasiperiodic systems.

58 Consider the embedding of the system into 2D. The atomic surface in the unit cell (the interval $[0,1])$ consists of a portion $c$ of 59 in up particles and a portion $(1-c)$ of spin down particles. Take the first in the interval $[0, c]$ and the second in the interval $[c, 1]$. At the 
Philosophical Magazine,

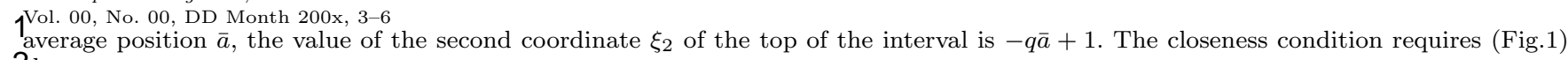
2hat 3

4

$-q \bar{a}+1=-c$.

5

Since $q=1 / b$ it follows that

7

8

9

$\frac{\bar{a}}{b}=1+c \rightarrow n_{2} / n_{1}=1+c$

19he physical reason for this condition is a condition on the charge. Because A atoms in the aperiodic chain are divalent and the B atoms 11tonovalent, charge neutrality requires that

12

15he concentration $c$ is the parameter $x$ in the chemical formula $A_{1+x}\left(A_{x}^{\prime} B_{1-x}\right) O_{3}$. Therefore, the charge condition leads to the closeness 16 ndition, which has a simple interpretation in the superspace picture. For periodic systems, this implies a rational value of $c$, but for an aperiodic system the approximants $n_{2} / n_{1}$ tend to $1+c$.

\section{Ground state}

21 he ground state of the system corresponds to the minimum of the potential energy $V$. This may be calculated numerically for finite 2. pproximants, using a relaxation procedure. The initial configuration then may be of importance, and should be varied with respect to 23number of parameters. For the spin wave which determines the distribution of the long intervals in chain 1, we take

24

$$
\sigma_{n}=\operatorname{Sign}[\sin (2 \pi m c n+\phi)-\cos (\pi c)]
$$

vonere $\phi$ determines the starting point of the spin distribution and $m$ the number of sign changes. The value $m=1$ corresponds to a distribution of spins on the atomic surface which consists of a connected region with value +1 and and its complement with value -1 . 2Phis gives a most non-uniform distribution of spins in the internal space, but the distribution in physical space is then as uniform as 298 ssible. On the other hand, a spin wave with one harmonic in physical space (non-uniform in this space) gives a spin distribution on The atomic surface which is very uniform. The value of $\phi$ determines the position of the spin wave with respect to the two chains. Special points would be $0, \pi / 2, \pi$ and $3 \pi / 2$, because then the number of spins up could be extremal.

31 The potential energy minima give the ground states for values of $n_{1}$ and $n_{2}$ such that $n_{2} / n_{1}$ is an approximation to $c$. These are 38. en embedded into two-dimensional space using the customary procedure. The $2 \mathrm{D}$ lattice coordinates $\xi_{1}$ and $\xi_{2}$ are given by

33

36 he point in physical space gives an infinite number of embedded points

37

For small values of $\lambda$ the atomic surfaces are smooth. For larger values of the interaction the atomic surfaces become discontinuous. For 40 ill stronger interaction (and $\Delta \neq 0$ ) the result is a modulated phase with one average lattice constant, and a fractal atomic surface. 41 For $\Delta=0, \lambda=0$ the atomic surfaces are straight lines parallel to the one of the two basis vectors. For the choice of basis made 42re, the particles of chain 1 fall on lines parallel to $V_{I}$. The atomic surface corresponding to chain 1 consists of alternative regions for 43 d 2 types of particles. Maximal separation in $V_{I}$ means that particles with spin up fall on $0<\xi_{2}<c$ and those with spin down on $c_{C}<\xi_{2}<1$. In direct space this distribution corresponds to a uniform distribution of spins. This means the following. If we have $n_{11}$ spins 44 , and $n_{12}$ spins down, we can plot the distribution on a square grid by going from $(0,0)$ to $\left(n_{11}, n_{12}\right)$ increasing the first coordinate 45 one when there is a spin up, and the second coordinate when there is a spin down. The uniform distribution corresponds to the path 46 at follows the straight line between begin and end point as closely as possible. The other extreme is obtained when $n_{11}$ spins up are fallowed by $n_{12}$ spins down. On the grid this gives a path consisting of two straight lines (one vertical, the other horizontal), and in 4 ifternal space it gives a uniform distribution of spins. Varying the distribution by varying the number of harmonics in the spin wave 48merical calculations lead to the conclusion that always the lowest energy per particle corresponds to the uniform distribution in real 49 ace, although the energy differences are small.

50 A second parameter is the relative phase of spin wave and modulation. The lowest energy is obtained for $\phi=\pi / 2$ if $\lambda>0$ and $50=3 \pi / 2$ if $\lambda<0$. For negative $\lambda$ this means that the closeness condition is satisfied, or in other words that the spin wave is uniform in 5 hysical space. Again the energy differences are small.

52 For $\Delta=0, \lambda \neq 0$ the lines are no longer straight, but a modulation of the chains sets in. For small values of the interaction 53 mameter the modulation is smooth. For larger values the atomic surface is split into disjoint parts.

If $\Delta \neq 0$, then the atomic surface consists of disjoint parts already from $\lambda=0$. There is an average slope of the pieces. For $\lambda=0$, 54 is slope is completely determined by the value of $\Delta$. For $\lambda \neq 0$ discontinuities in the disjoint parts set in. For large values of $\lambda$ the 56 omic surfaces of both chains merge. The resulting structure is a modulated structure with displacive and occupation modulation. The $56^{n b e d d i n g ~ s h o w s ~ p a r a l l e l ~ a t o m i c ~ s u r f a c e s, ~ w i t h ~ d i s c o n t i n u i t i e s ~ a n d ~ a l t e r n a t i v e ~ r e g i o n s ~ o f ~ p a r t i c l e s ~ o f ~ b o t h ~ c h a i n s . ~}$

57 Because the energy differences between uniform and non-uniform distributions are small, there are many metastable states. The 57on-uniform distributions correspond to atomic surfaces with an increasing number of gaps if the number of harmonics in the spin wave 58 creases. Nevertheless, the gaps in one atomic surface correspond to pieces in the atomic surfaces of the neighbours, but the closeness 59 ndition is no longer satisfied. 
Philosophical Magazine,

$1^{\text {Vol. 00, No. 00, DD Month 200x, 4-6 }}$

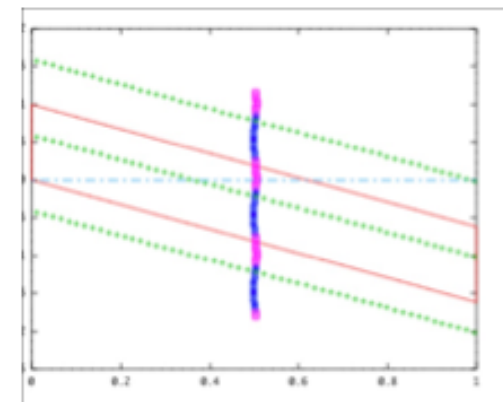

Weak int., uniform $\Delta=0$

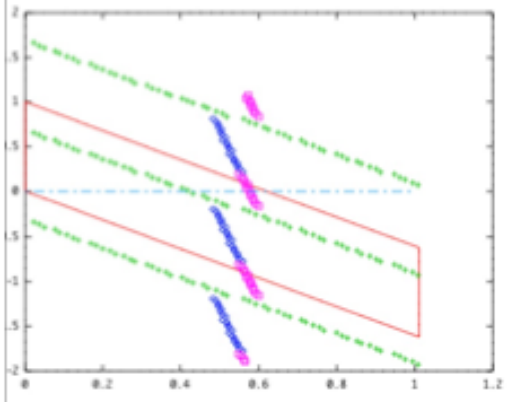

$\Delta=0.05$, weakly uniform

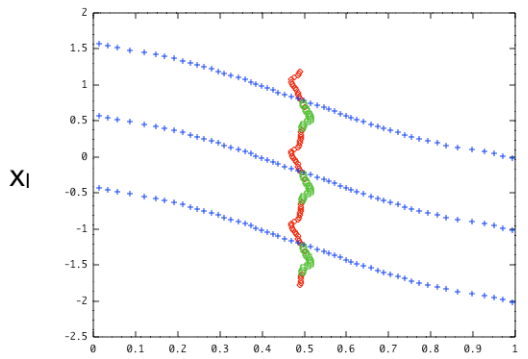

XE

Figure 2. Embedding of a solution with $\lambda=-1.2, \Delta=0$.

499 9 gure 3. Embedding of 5 solutions. In the first row the pictures give the difference between uniform spin distributions in physical and 50 internal space. For strong coupling $\lambda$ the atomic surfaces will become discontinuous for $\Delta=0$ as well. For $\Delta>0$ there is always a 51 discontinuity (frame 4). In the second row the changes in the atomic surfaces are shown if the spin wave is taken to be weakly or

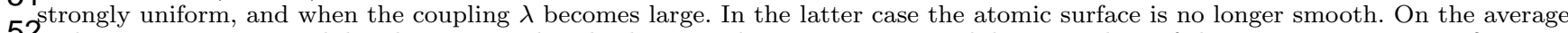
52 there remains one modulated system with a displacive and an occupation modulation. Values of the parameters: $\alpha=1.0, \beta=1.5$, $\bar{a} / b=34 / 55$. 58 here is no sign of lock-in terms in the the energy. However, when the interaction parameter $\lambda$ becomes stronger, there is a weak lock-in 5 ffect for $c=0.5$. (Cf. Fig.4) 
Philosophical Magazine,

$1^{\text {Vol. 00, No. 00, DD Month 200x, 5-6 }}$

2

3

4

5

6

7

8

9

10

11

15 Figure 4. Energy per particle for the ground state as function of the composition $c . \lambda=0.25, \Delta=0.05, n_{2}=84$. The vertical lines

\section{Dynamics}

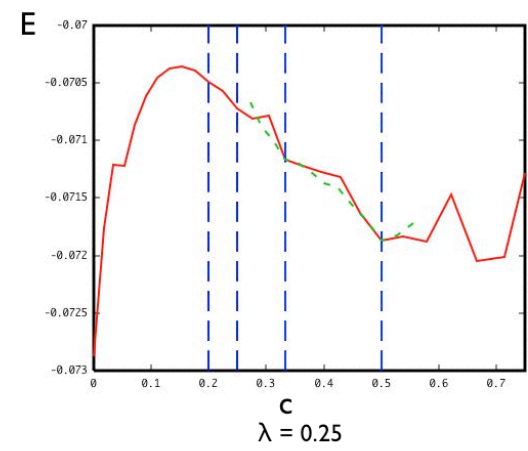

2 Phe vibrational spectrum of an aperiodic system may be obtained from the dispersion curves of its approximants. For an approximant 21 he frequencies are the roots of the eigenvalues of the equations

$$
m_{n} \omega^{2} u_{i}=\left.\sum_{j} \frac{\partial^{2} V}{\partial x_{i} \partial x_{j}}\right|_{\mathrm{eq}} u_{j}
$$

Eqr the APDC there is always a zero frequency vibrational mode, because of translation invariance. For an incommensurate phase in the $25 \mathrm{CM}$ there may be a second such mode as well, if the modulation function is smooth. This corresponds to smoothly connected atomic 28\&rfaces. This happens for the APDC only when $\Delta=0$. One expects that for the latter case, there is a zero frequency phason mode as $29 \mathrm{ng}$ as the modulation is smooth. And for $\Delta \neq 0$, one expects always a phason gap. This is confirmed by the calculations. If $\Delta \neq 0$, there 30 only a single mode with zero frequency, and there is always a phason gap as soon as $\lambda \neq 0[3]$.

31. For $\lambda=0$ the eigenmodes are limited to one of the chains. If $\lambda \neq 0$, then still eigenmodes may be concentrated on one of the 3 hains, but there are excitations involving both chains in a substantial way. An example is given by the zero frequency mode. If $\lambda=0$ 3Rere are two of such modes, and every linear superposition of the two is an eigenmode. If $\lambda \neq 0$, then this degeneracy is broken. The 3 3 perposition of the two modes in the separate chains which has an equal displacement for every particle remains with zero frequency. nnother combination describes a relative shift of both chains, and has non-zero frequency. This situation occurs again for every crossing 34tint of the dispersion curves of the separate chains, in the extended zone scheme.

35 The phason mode with wave vector zero is the phonon mode for which the displacement of the centres of mass of the two 36bsystems, with respect to each other, is maximal. The eigenvector of this phason mode, when there is a phason gap. has maximal displacements of the atoms near the discontinuity of the modulation function. This has been found also in the DIFFOUR (discrete 37 ustrated $\phi^{4}$ ) model for incommensurate phases [4].

38 A shift of the subsystems with respect to each other, which may also be described as a change of the internal coordinate in 39 perspace, connects ground states of the same energy. This is, however, not a harmonic excitation, because it involves jumps of atoms lotween positions. These jumps, in quasicrystals called phason jumps, are actually non-linear excitations. It has been shown for the DCM that such a shift of one system with respect to the other does not cost energy if the atomic surfaces are continuous AND the velocity is 4jelow a certain threshold [3]. In this case, however, the atomic surfaces are discontinuous, which means that a phason shift is dissipative.

\section{Concluding remarks}

46

47 simple model has been presented for a class of composition flexible compounds. It is a generalisation of the double chain model for incommensurate composites. Although there is an additional length scale, the rank of the system remains two, because of charge 48 utrality. The embedding in superspace consequently is two-dimensional.

49 If the value of the parameter $\Delta$ is non-zero, the embedding is always discontinuous. In the 4 dynamics this leads to the presence 50 a phason gap. There is no dissipationless motion of one subsystem with respect to the other. The motion is pinned.

51 The model shows a transition from a simple composite system (for $\Delta=0$ ) to a system that is more easily described as a combination of displacive and occupation modulation, with non-crossing atomic surfaces. This is another proof that modulated phases 52ad composites are not fundamentally different. The fact that for $\Delta \neq 0$ the atomic surfaces are bounded, and the system becomes 58 milar to a quasicrystalline structure. The three classes (modulated phases, composites and quasicrystals) are not mutually exclusive.

54 There is no indication for a preference for commensurate compositions. The energy per particle is a smooth function, without 54 sharp minima, of the concentration. The lowest energy is obtained for an alteration of prisms and octahedra which is as homogeneous as 5 Sossible.

56 A straightforward generalisation to two dimensions consists of an array of parallel chains. Particles with spin of type A have an 5 mmodulated basis structure with positions

$$
x_{n, \ell}=x_{n-1}+a+\sigma_{n} \Delta, \quad y_{n, \ell}=\ell c,
$$

Philosophical Magazine

ISSN 1478-6435 print/ISSN 1478-6443 online (c) 200x Taylor \& Francis

http://www.tandf.co.uk/journals

DOI: $10.1080 / 1478643 Y$ Yxxxxxxxx 
Philosophical Magazine, 1'Vol. 00, No. 00, DD Mnnth $90 n v 6-6$

2

3

4

5

6

7

8

9

10

11

12

13

14

15

16

17

18

19

20

21

22

23

24

25

26

27

28Figure 5. Dispersion curves in the Brillouin zone of the approximant structure, for $\Delta \neq 0$, for 4 values of $\lambda$. Upper row, left: $\lambda=0$ : 29 the are 2 modes with frequency zero. For increasing values of $\lambda$ the phason gap opens. Simultaneously gaps open at the Brillouin zone 30

31 d the particles in subsystem 2 have basic structure positions

32

33

34

35

36

37

38

39

40

41

4 bhis higher-dimensional model is under investigation.

43

44

\section{3eference}

46] L. Elcoro, J.M. Perez-Mato, J. Darriet, and A. El Abed. Superspace description of trigonal and orthorhombic $\mathrm{A}_{1+x} \mathrm{~A}_{x} \mathrm{~B}_{1-x} \mathrm{O}_{3}$ 47 compounds as modulated layer structures; application to the refinement of trigonal $\mathrm{Si}_{6} \mathrm{Rh}_{5} \mathrm{O}_{15}$. Acta Cryst. B, 59:217-233, 2003.

4[] M. Evain, F. Boucher, O. Gourdon, V. Petricek, M. Dusek, and P. Bezdicka. Incommensurate versus commensurate description of the $49 \mathrm{~A}_{x} \mathrm{BX}_{3}$ hexagonal perovskite-type structure. $\mathrm{Sr}_{1.2872} \mathrm{NiO}_{3}$ incommensurate composite compound example. Chem. Mat., 10:3068-376,
1998 .

50] T. Janssen, O. Radulescu, and A.N. Rubtsov A.N. Phasons, sliding modes and friction. Eur.Phys.J., B29:85-95, 2002.

5f] T. Janssen and J.A. Tjon. Microscopic model for incommensurate crystal phases. Phys. Rev., 25:3767-3785, 1982.

55 J.M. Perez-Mato, M. Zakhour-Nakl, and J. Darriet. Structure of composites $\mathrm{A}_{1+x}\left(\mathrm{~A}^{\prime}{ }_{x} \mathrm{~B}_{1-x}\right) \mathrm{O}_{3}$ related to the $2 \mathrm{H}$ hexagonal perovskite: 52 relation between composition and modulation. J. Chem. Materials, 9:2795-2808, 1999.

58] O. Radulescu and T. Janssen. Sliding mode and breaking of analyticity in the double chain model of incommensurate composites. 54 Phys. Rev. B, 60:12737-12745, 1999.

55 T. van Erp, A. Fasolino, O. Radulescu, and T. Janssen. Pinning and phonon localization in frenkel-kontorova models on quasiperiodic 56 substrates. Phys. Rev. B, 60:6522-6528, 1999. 